\title{
Structure of Free Radical in $\gamma$-Irradiated 21-Hydroxyprogesterone (Deoxycorticosterone) Single Crystals. ESR/ENDOR and DFT Studies
}

\author{
A. SzyczeWski ${ }^{a, *}$, J. Pietrzak ${ }^{a}$ And K. MöBius ${ }^{b}$ \\ ${ }^{a}$ Institute of Physics, A. Mickiewicz University \\ Umultowska 85, 61-614 Poznań, Poland \\ ${ }^{b}$ Institute of Molecular Physics, Free University of Berlin \\ Arnimallee 14, 14195 Berlin, Germany
}

\begin{abstract}
Single crystals of 21-hydroxyprogesterone have been $\gamma$-irradiated at $295 \mathrm{~K}$ and studied using X-band ESR and ENDOR. The structure of the one type radical has been determined on the basis of an analysis of the angular variation of the spectra. This radical is formed by abstraction of the hydrogen atom from the $\mathrm{C}(6)$ carbon, while the unpaired electron is delocalised onto the system $\mathrm{O}(3), \mathrm{C}(3), \mathrm{C}(4), \mathrm{C}(5), \mathrm{C}(6)$. Hyperfine splitting constants and unpaired electron density distribution have been calculated for the proposed radical structure by using the Gaussian98 set of programs. The results are in very good agreement with the experimental data. The effect of the hydrogen bond and biological activity on the anisotropy of $\alpha$-hyperfine splitting tensor have also been discussed.
\end{abstract}

PACS numbers: 76.30.--v, 76.70.-r, 78.70.--g, 31.15.Ew

\section{Introduction}

In order to fully understand chemical and biochemical reactions it is essential to determine the electronic and geometric structures of the molecules involved. One of the most important groups of biologically active natural compounds are the steroids. The parent compound from which all the steroid hormones are

*corresponding author; e-mail: aszy@amu.edu.pl 
ultimately derived is cholesterol. The group of steroids known as the corticosteroids are formed in adrenal cortex and are usually classified under two headings: (a) the 17-hydroxylates corticosteroids and (b) the 17-deoxycorticosteroids, depending on the presence or absence of a $17 \alpha$-hydroxyl group. The title compound 21-hydroxyprogesterone, known as deoxycorticosterone (DOC) (Fig. 1) belongs to the other class - mineralocorticoids. In contrast to glucocorticoids, the activity of mineralocorticoids, such as aldosterone or DOC, is confined to effects on salt and water metabolism.

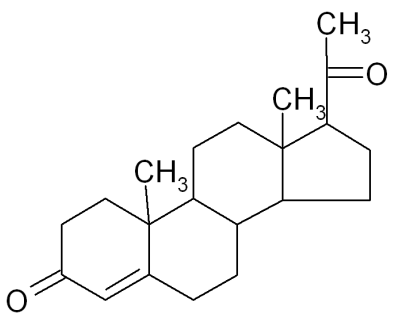

21-OH progesterone

Fig. 1. The structure of 21-hydroxyprogesterone.

Rexroad and Gordy [1] were the first to report on ESR study of free radicals in X-ray irradiated powdered cholesterol. Recently, intense ESR and ENDOR [2-4] studies have been undertaken to elucidate the structure of free radicals formed in $\gamma$-irradiated single crystals of selected steroids. The analysis of hyperfine coupling tensors enables an accurate determination of the geometry of the radical formed and the degree of conformational changes after the process of radical formation. The presence of water molecule as well as the hydrogen bond were found to be the factors influencing the type of radical formed. ENDOR provides a better resolving power of the spectra than ESR and enables simultaneous identification of a greater number of radicals.

We have chosen to study 21-hydroxyprogesterone which contains a hydroxyl group in its structure, but no intermolecular hydrogen bond has been detected in this compound by crystallographic methods [5]. The analysis of its crystallographic data shows a possibility of an intramolecular hydrogen bond formation by the atoms $\mathrm{C}(20), \mathrm{C}(21), \mathrm{O}(21), \mathrm{H}(21)$, and $\mathrm{O}(20)$.

Density functional theory (DFT) calculations have been performed in order to compare experimental and calculated geometries and the values of spin Hamiltonian parameters. In order to obtain additional information on the type of hydrogen bond, a DOC study was performed by IR spectroscopy, for a polycrystalline sample and a sample dissolved in a non-polar solvent. An attempt was made to establish a relation between the spectroscopic parameters and biological activity. 


\section{Experimental}

A sample of deoxycorticosterone corticosterone (4-pregnen-21-ol-3,20-dione) $\left(\mathrm{C}_{21} \mathrm{H}_{30} \mathrm{O}_{3}\right)$ of high purity was a gift from Schering AG Berlin. The crystal and molecular structure have been determined by Dideberg, Campsteyn, and Dupont [6].

The single crystals were $\gamma$-irradiated with a ${ }^{60} \mathrm{Co}$ source at $295 \mathrm{~K}$ with a total dose of up to $150 \mathrm{kGy}$. ESR and ENDOR spectra were taken at X-band microwave frequencies. ESR spectra were recorded using $100 \mathrm{kHz}$ modulation on an X-band VARIAN and additionally on a RADIOPAN (Poland) ESR spectrometers. The ENDOR spectrometer has been described elsewhere [7].

All DFT geometry optimizations were performed with the B3LYP hybrid functional in Pople's 6-31G $(d)$ basis set, using the atoms' coordinates from the crystal structure [6] modified to correspond to the radical structure, i.e. with axial hydrogen atom $\mathrm{H}(6 a)$ removed. The B3LYP functional is a combination of Becke's three-parameter hybrid exchange functional [8] and the Lee-Yang-Parr correlation functional [9] are included in the set of programs Gaussian 98 [10].

The optimised structure was used for single point calculations at the B3LYP level employing different basis sets: 6-31G $(d), 6-31 \mathrm{G}(d, p), 6-311 \mathrm{G}(d)$, $6-311 \mathrm{G}(d, p)$, and EPR-II [11-13].

\section{Results and discussion}

ESR spectra of $\gamma$-irradiated 21-hydroxyprogesterone (deoxycorticosterone) shown in Fig. 2, have a complex structure and, in some orientations, are poorly resolved. Prominent lines in the ESR spectrum can be interpreted as two doublets, each of which is split into a triplet. The spectrum is slightly asymmetrical, which suggests the presence of more than one type of radicals. Figure 3 presents typical ENDOR spectra of DOC. A comparison of ESR and ENDOR spectra proves that the triplet is really a double doublet of close lying values of hyperfine couplings. The ENDOR spectra proves the presence of radical $I$.

The tensors describing four hyperfine interactions and the $g$-factor of radical $I$ were calculated and their values are collected in Table I. The values of the hyperfine splitting constants and their amplitudes indicate the occurrence of two $\alpha$-hyperfine splitting constants of close lying values and two $\beta$-hyperfine splittings. The analysis of these data leads to a conclusion that radical $I$ is formed by abstraction of a hydrogen atom from the carbon atom $\mathrm{C}(6)$ with the unpaired electron delocalised onto $\mathrm{C}(3), \mathrm{C}(4), \mathrm{C}(5), \mathrm{C}(6)$, and $\mathrm{O}(3)$ (Fig. 4).

The $\pi$-spin density of carbons adjacent to $\alpha$-protons are calculated from hyperfine constant (hfc) values by using the McConnell equation [14], based on $Q$ value of $-72 \mathrm{MHz}$ :

$$
\boldsymbol{A}_{\text {iso }}^{\alpha}=\boldsymbol{Q}_{\mathrm{H}} \rho_{\mathrm{C}}^{\pi}
$$



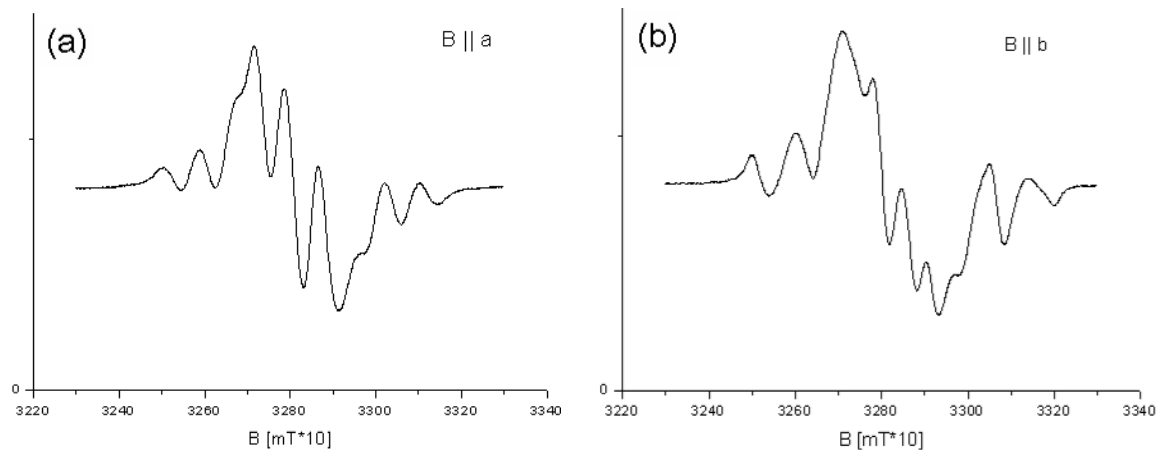

Fig. 2. ESR spectra of $\gamma$-irradiated 21-hydroxyprogesterone single crystal, recorded at room temperature for $H$ parallel to axis $a$ (a) and $b(\mathrm{~b})$.

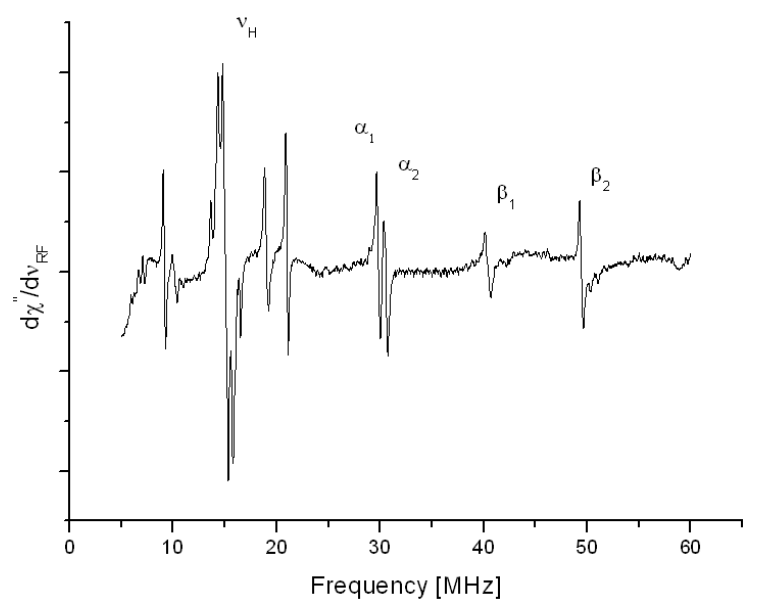

Fig. 3. ENDOR spectrum of $\gamma$-irradiated 21-hydroxyprogesterone single crystal for $H$ parallel to axis $b$.

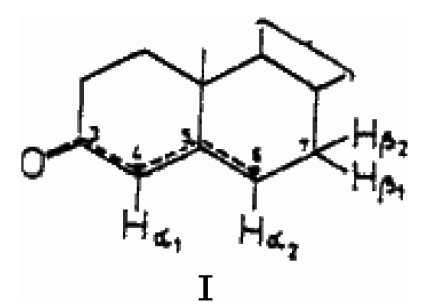

Fig. 4. Structure of radicals $I$ formed a $\gamma$-irradiated 21-hydroxyprogesterone single crystal.

and the observed $\alpha$-splittings for the spin density distribution is 0.44 on $\mathrm{C}(6)$ and 0.43 on $\mathrm{C}(4)$. For $\beta$-protons the Heller-McConnell equation [15] is used

$$
\boldsymbol{A}_{\text {iso }}^{\beta}=\left(B_{1}+B_{2} \cos ^{2} \Theta\right) \rho_{\mathrm{C}}^{\pi},
$$


TABLE I

$\boldsymbol{g}$-Tensor and principal values of hyperfine coupling tensors and direction cosines for radical $I$ in a $\gamma$-irradiated single crystal of 21-hydroxyprogesterone.

\begin{tabular}{c|c|r|r|r|c}
\hline \hline Tensor & $\begin{array}{c}\text { Principal values }^{a} \\
{[\mathrm{Mhz}]}\end{array}$ & \multicolumn{3}{|c|}{ Direction cosines } & $\varphi[\mathrm{deg}]$ \\
\hline $\boldsymbol{g}$ & 2.00257 & 0.4252 & -0.9049 & -0.0161 & $18.6^{c}$ \\
& 2.00397 & -0.9044 & -0.4242 & -0.0463 & \\
& 2.00540 & -0.0351 & -0.0342 & 0.9998 & \\
\hline $\boldsymbol{A}_{\alpha 1}$ & -15.06 & -0.8510 & -0.2235 & 0.4753 & $4.6^{b}$ \\
& -30.53 & 0.2184 & -0.9736 & -0.0666 & $6.4^{c}$ \\
& -48.89 & -0.4776 & -0.0471 & -0.8773 & \\
\hline $\boldsymbol{A}_{\alpha 1}$ (iso) & $-30.83(31.40)^{*}$ & & & \\
\hline $\boldsymbol{A}_{\alpha 2}$ & -15.86 & -0.8463 & -0.2541 & 0.4682 & $6.1^{b}$ \\
& -31.90 & 0.3224 & -0.9448 & -0.0705 & $4.9^{c}$ \\
& -47.78 & -0.4240 & -0.2106 & -0.8808 & \\
\hline $\boldsymbol{A}_{\alpha 2}$ (iso) & $-31.82(32.66)^{*}$ & \multicolumn{3}{|c|}{} & \\
\hline $\boldsymbol{A}_{\beta 1}$ & 46.43 & -0.3203 & -0.8964 & -0.3065 & \\
& 59.4456 .17 & 0.9232 & -0.3678 & 0.1109 & \\
& $62.64(58.87)^{*}$ & 0.2122 & 0.2474 & -0.9454 & \\
\hline $\boldsymbol{A}_{\beta 2}$ & 66.22 & -0.9202 & -0.0431 & 0.3890 & \\
& 71.4370 .87 & 0.2515 & 0.8267 & 0.5033 & \\
& $74.96(73.77)^{*}$ & -0.2999 & -0.5610 & -0.7716 & \\
\hline
\end{tabular}

${ }^{a}$ eigenvalues $+/-0.04[\mathrm{Mhz}]$,

${ }^{b}$ angle to the direction of $\mathrm{C}(4)-\mathrm{H}(4)$ bond,

${ }^{c}$ angle to the normal to the plane $\mathrm{C}(4) \mathrm{C}(5) \mathrm{C}(6)$,

*B3LYP 6-311G $(d, p)$ calculated values.

where $B_{1}$ is usually assumed to be zero, $B_{2}=50 \mathrm{G}$ and $\Theta$ is the torsional angle between the $p_{z}$ orbital and $\mathrm{C}-\mathrm{H}_{\beta}$ direction.

The hyperfine splitting coupling values, theoretically calculated using DFT methods and their experimental values are presented in Table I.

Comparing the hydrogen hyperfine splitting constant (hfsc) at the B3LYP/ $6-31 G(d)$ level of theory with the experimental results already shows a reasonable agreement. The best agreement of the isotropic values is obtained for the results obtained assuming the basis set $6-311 \mathrm{G}(d, p)$.

The differences between the calculated and experimental isotropic values of hydrogen atoms $\mathrm{H} 4$ and $\mathrm{H} 6$ are small in the order of magnitude of $0.5 \mathrm{MHz}$ and circa 1.5 MHz for $\mathrm{H} 7 \mathrm{a}$, for both isotropic and anisotropic values.

The calculated $\rho_{\mathrm{C}}^{\pi}$ values have been used for estimation of $\beta \mathrm{hfs}$, using the Heller-McConnell equation [15]. Making use of calculated values of the torsion 
angles $\mathrm{H}(6) \mathrm{C}(6) \mathrm{C}(7) \mathrm{H}(7 \mathrm{a})$ and $\mathrm{H}(6) \mathrm{C}(6) \mathrm{C}(7) \mathrm{H}(7 \mathrm{~b})$ the values $\Theta_{\beta 1}=41.2^{\circ}$ and $\Theta_{\beta 2}=154.5^{\circ}$ as well as $A_{\beta 1}=70.88 \mathrm{MHz}$ and $A_{\beta 2}=56.50 \mathrm{MHz}$ have been calculated. The calculated values are in excellent agreement with the experimental data.

Radical $I$, as mentioned previously, is formed by abstraction of an axial hydrogen atom $\mathrm{H}(6 \mathrm{~A})$ from the carbon atom at position 6 . The formation of the radical is connected with atom displacement, particularly $\mathrm{C}(6)$, as a result of hybridization change from $s p^{3}$ into $s p^{2}$, i.e. causing transfer of $\mathrm{C}(6)$ from the tetragonal structure to the planar one. Identification of radicals by ESR/ENDOR as well as determination of molecular changes induced by irradiation are based on a proper correlation between the directions of the principal tensor elements and the related directions of the undamaged molecule. The position and orientation of the interacting $\alpha$-proton is obtained from the direction of the minimum $\alpha$-hfs constant and the direction of $2 p_{z}$ orbital is obtained from the intermediate $\alpha$-splitting. This direction can also be obtained from the minimum $\boldsymbol{g}$-tensor value. The direction of the $2 p_{z}$ orbitals of the $\mathrm{C}(4)$ and $\mathrm{C}(6)$ atoms should be normal to the plane spanned by carbon atoms $\mathrm{C}(3), \mathrm{C}(4), \mathrm{C}(5)$, and $\mathrm{C}(6)$ and the $\mathrm{C}_{\alpha}-\mathrm{H}_{\alpha}$ direction close to $\mathrm{C}(4)-\mathrm{H}(4)$. A comparison of the crystallographic directions with experimental data is presented in Table I, and gives a good agreement.

Earlier [2] an attempt was made to analyse the effect of progesterone skeleton substitution by hydroxyl groups (corticosteroids are very often the multi-hydroxyl substituted progesterons) on the anisotropy of hfs tensors. Examination of the anisotropy of the $\alpha$-hyperfine splitting in radical $I$, defined as $A_{z}-A_{x} / A_{z}$ (fractional value of anisotropy) was performed, where $A_{x}$ and $A_{z}$ are the minimum and maximum principal values of the $\alpha$-hfs. Table II presents the hyperfine anisotropy values compared with the unpaired electron density and the length of the corresponding hydrogen bond for a series of steroid compounds: progesterone, cholest-4-en-3-one, $17 \alpha$-hydroxyprogesterone, 17 $\alpha, 21$-dihydroxyprogesterone and 21-hydroxyprogesterone.

To find out whether values of the $\Delta A / A_{z}$ parameter are related to the biological activity of a given compound, a correlation between this parameter and the rate constant $1 / K$ has been analysed for hydroxy substituted corticostreroids in the reactions with corticosteroid binding globulin (CBG) and testosterone binding globulin (TBG) [16]. TBG has been recently called sex hormone binding globulin (SHBG). These two steroid-binding proteins, corticosteroid-binding globulin, and sex hormone binding (or TBG) globulin circulate in plasma. They both have several different but related physiologic functions. Each is a major determinant of the concentration of the physiologically important hormones that they bind. CBG regulates the concentration of free cortisol and progesterone, and SHBG regulates the concentration of free testosterone, dihydrotestosterone, and, to a lesser extent, estradiol. It is this small free fraction of the appropriate hormone that is the active base affecting the hormone activity. In the past few years, it has been shown 
TABLE II

Anisotropy parameters of hyperfine couplings, $\Delta A / A_{z}$, unpaired electron density $(\rho)$, and the length of hydrogen bonds for selected steroid compounds.

\begin{tabular}{|c|c|c|c|c|c|}
\hline \multirow[t]{2}{*}{ Compound } & \multirow{2}{*}{$\begin{array}{c}A_{\alpha} \text { (iso) } \\
{[\mathrm{Mhz}]}\end{array}$} & \multirow[t]{2}{*}{$\Delta A / A_{z}$} & \multicolumn{2}{|c|}{ Hydrogen bond lengths $[\AA]$} & \multirow[t]{2}{*}{$\rho$} \\
\hline & & & $\mathrm{O}(3)-\mathrm{O}(17)$ & $\mathrm{O}(3)-\mathrm{O}(21)$ & \\
\hline \multirow[t]{2}{*}{ Progesterone[2] } & (1) $31.12^{a}$ & 0.670 & & & 0.432 \\
\hline & (2) $31.91^{b}$ & 0.679 & & & 0.443 \\
\hline \multirow[t]{2}{*}{ Cholest-4-en-3-one [2] } & (1) $31.73^{a}$ & 0.668 & & & 0.441 \\
\hline & (2) $32.25^{b}$ & 0.680 & & & 0.448 \\
\hline \multicolumn{6}{|l|}{ 17-hydroxy- } \\
\hline progesterone [2] & $30.80^{c}$ & 0.710 & 2.79 & - & 0.428 \\
\hline $17 \alpha, 21$-dihydroxy- & (1) $30.46^{a}$ & 0.687 & 3.07 & 2.84 & 0.423 \\
\hline progesterone [3] & (2) $32.07^{b}$ & 0.705 & 3.07 & 2.84 & 0.445 \\
\hline 21-hydroxy- & (1) 31.24 & 0.679 & - & - & 0.429 \\
\hline progesterone & (2) 31.82 & 0.668 & - & - & 0.442 \\
\hline
\end{tabular}

that both these proteins have a high affinity to specific receptors on the plasma membranes of a variety of cells. It has also been shown that when SHBGs binding sites are occupied it cannot bind to its receptor; only unliganded SHBG can.
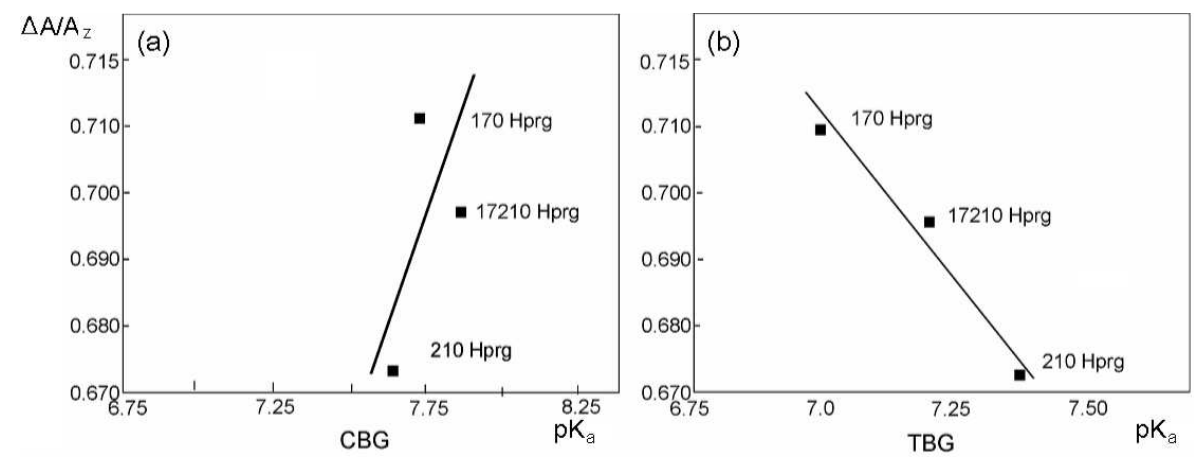

Fig. 5. Anisotropy $\left(\Delta A / A_{z}\right)$ of the hyperfine splitting (hfs) tensor versus $p K_{a}$ (CBG) (a) and TBG (b).

Figure 5 presents the anisotropy of the hfs tensor versus the reaction constants characterising the reactions of steroids with TBG and CBG $(\log (1 / K)=$ $\left.p K_{a}[15]\right)$, a linear character of the dependence is clearly seen.

The results indicate that the anisotropy of the hyperfine splitting tensor $\alpha$ increases with increasing strength of the hydrogen bond and with increasing hor- 
mone activity of sex hormone binding globulin and corticosteroid binding globulin. As the hydrogen bond is strongly directional, it causes a polarisation of the electronic structure of ring $\mathrm{A}$ of the steroid skeleton. The interaction of steroids with transporting proteins also has a hydrogen bond nature and an increase in its strength seems to be directly related to the $p K_{a}$ values of this reactions.

\section{Acknowledgments}

The calculation were performed in Supercomputer Network Center (PCSS, Poznań/Poland) which is highly acknowledged.

\section{References}

[1] H.N. Rexroad, W. Gordy. Proc. Nat. Acad. Sci. USA 45, 256 (1959).

[2] A. Szyczewski, Applied Radiation \& Isotopes 47, 1675 (1996).

[3] A. Szyczewski, K. Möbius, J. Mol. Struct. 318, 87 (1994).

[4] A. Szyczewski, B. Endeward, K. Möbius, Applied Radiation ES Isotopes 49, 59 (1998).

[5] W. Duax, Atlas of Steroid Structure, Plenum Press, New York 1985.

[6] O. Dideberg, H. Campsteyn, L. Dupont, Acta Crystallogr. B 29, 103 (1973).

[7] K. Möbius, Magn. Reson. Rev. 12, 285 (1987).

[8] A.D. Becke J. Chem. Phys. 98, 1372 (1993).

[9] C. Lee, W.Yang, R.G. Parr, Phys. Rev. B 37, 785 (1988).

[10] M.J. Frisch, G.W. Trucks, H.B. Schlegel, G.E. Scuseria, M.A. Robb, J.R. Cheeseman, V.G. Zakrzewski, J.A. Montgomery, R.E. Stratmann, J.C. Burant, S. Dapprich, J.M. Millam, A.D. Daniels, K.N. Kudin, M.C. Strain, O. Farkas, J. Tomasi, V. Barone, M. Cossi, R. Cammi, B. Mennucci, C. Pomelli, C. Adamo, S. Clifford, J. Ochterski, G.A. Petersson, P.Y. Ayala, Q. Cui, K. Morokuma, D.K. Malick, A.D. Rabuck, K. Raghavachari, J.B. Foresman, J. Cioslowski, J.V. Ortiz, B.B. Stefanov, G. Liu, A. Liashenko, P. Piskorz, I. Komaromi, R. Gomperts, R.L. Martin, D.J. Fox, T. Keith, M.A. Al-Laham, C.Y. Peng, A. Nanayakkara, C. Gonzalez, M. Challacombe, P.M.W. Gill, B.G. Johnson, W. Chen, M.W. Wong, J.L. Andres, M. Head-Gordon, E.S. Replogle, J.A. Pople, Gaussian 98 (Revision A.7), Gaussian Inc., Pittsburgh (PA) 1998.

[11] V. Barone, in: Recent Advances in Density Functional Methods, Part I, Ed. D.P. Chong, World Scientific Publ. Co., Singapore 1996.

[12] N. Rega, M. Cossi, V. Barone, J. Chem. Phys. 105, 11060 (1996).

[13] V. Barone, Chem. Phys. Lett. 262, 201 (1996).

[14] R.W. Fessenden, R.H. Schuler, J. Chem. Phys. 39, 2147 (1963).

[15] H.M. McConnell, D.B. Chesnut, J. Chem. Phys. 28, 107 (1958).

[16] J.F. Dunn, B.C. Nisula, D. Rodbard, Clin Endocrinol. Metab. 53, 58 (1981). 